\title{
Tháng 8-2020, ISR với Nature, RePEc và Springer
}

\author{
Hồ Mạnh Toàn \\ Trung tâm ISR \& SDAG Lab \\ Trường Đại học Phenikaa
}

Hà Nội, 7-8-2020

ISR | Phenikaa Uni (URL: https://isr.phenikaa-uni.edu.vn/chitiet/tin-

tuc_2019_2 22 7 _58_84/thang-8-2020-isr-voi-nature-repec-va-springer) - COVID-19 bùng phát lần 2 đang gây lo lắng ở Việt Nam. Ảnh hưởng tiêu cực của dịch bệnh có thể cảm nhận được rất rõ trong công việc. Ngoài cản trở và đình trệ với sản xuất, công việc biên tập bình duyệt cũng bị tồn đọng, dẫn đến chờ đợi kéo dài. Tỷ lệ từ chối cũng có khuynh hướng tăng vọt.

Trong bối cảnh tiêu cực, cũng có vài điểm dưới đây giúp tinh thần đội ngũ sảng khoái hơn.

Cuối tháng 7, tạp chí của tổ hợp xuất bản học thuật Springer Nature về khoa học xã hội có tên SN Social Sciences (ISSN 2662-9283) đã có lời mời GĐ ISR Vương Quân Hoàng tham gia ban biên tập.

Lời mời đã nhận được sự đồng ý, và trang web tạp chí cũng cập nhật thông tin về đại diện ISR (Phenikaa Uni) trong ban biên tập (hình 1).

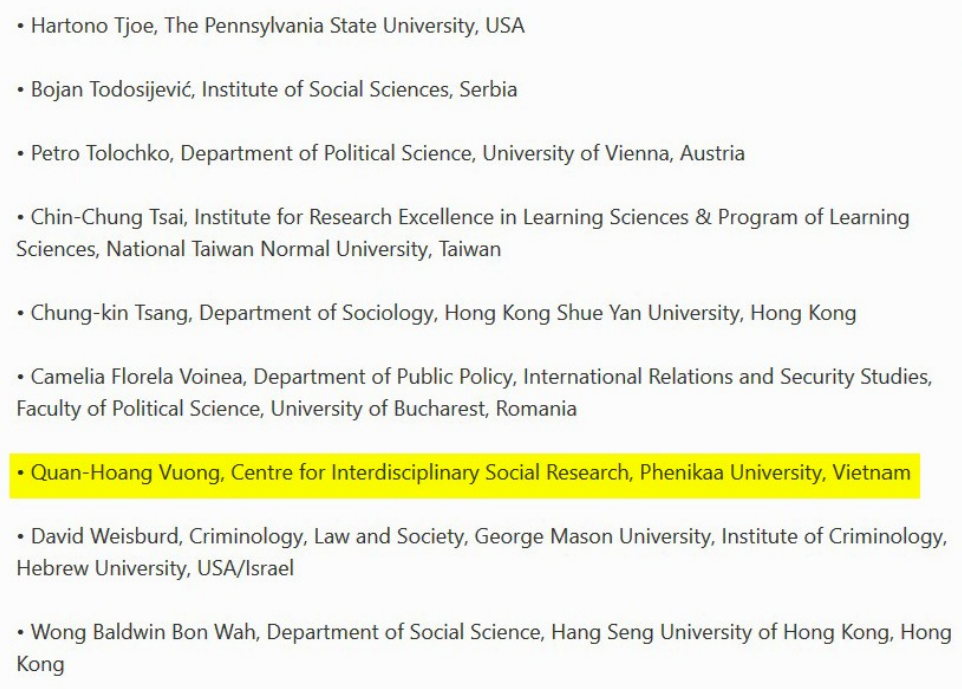

Springer

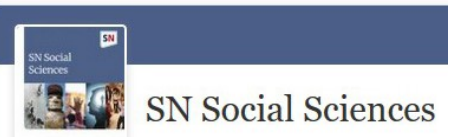

Hình 1. Đại diện ISR Phenikaa Uni trong BBT Springer Nature Social Sciences 
Ngay sau khi nhận lời, một số công việc thuộc về trách nhiệm biên tập đầu tiên đã bắt đầu được giao phó và tiến hành.[1]

Kết thúc tháng 7-2020, dữ liệu IDEAS/RePEc cũng cho thấy ISR vẫn giữ vững vị trí thứ 4 trong các đơn vị nghiên cứu-đào tạo kinh tế-quản trị ở nước ta. Trích vài đơn vị tốp trên của danh sách trong hình 2.

https:/ideas.repec.org/top/top.vietnam.html

(j)IDEAS Printed from https://deas.repec.org/top/top.vietnam.html

Top 25\% Institutions and Economists in Viet Nam, as of July 2020

\begin{tabular}{|c|c|c|c|c|c|c|c|c|}
\hline \multicolumn{2}{|c|}{ Explanations } & Institutions & Institutions $10 / 210$ & Authors & Authors 10 & Mo & & \\
\hline \multicolumn{9}{|c|}{ The rankings } \\
\hline \multicolumn{9}{|c|}{ Top $25 \%$ institutions in Viet Nam, all authors, all publication years } \\
\hline \multicolumn{9}{|c|}{ For Viet Nam, there are 133 authors affiliated with 49 institutions. All institutions in this region. } \\
\hline \multicolumn{2}{|c|}{ RankW.Rank } & \multicolumn{4}{|l|}{ Institution } & \multicolumn{2}{|c|}{ ScoreAuthors } & $\begin{array}{l}\text { Author } \\
\text { shares }\end{array}$ \\
\hline 1 & [2] & $\begin{array}{l}\text { Mekong Develc } \\
\text { Hanot, Vet Nam }\end{array}$ & oment Research Ins: & & & 2.27 & 9 & 8.5 \\
\hline 2 & [1] & $\begin{array}{l}\text { Unlversity of EC } \\
\text { Ho Chi Minh Cly, Vlet }\end{array}$ & onomlcs Ho Chi Min & City & & 2.3 & 11 & 10 \\
\hline \multirow[t]{2}{*}{3} & [4] & $\begin{array}{l}\text { Forelgn Trade L } \\
\text { Hanot, viet Nam }\end{array}$ & nlversity & & & 2.72 & 9 & 7.9 \\
\hline & & Centre for Inter & Isciplinary Social $R$ & iearch.Phe & ikaa & & & \\
\hline \multirow[t]{2}{*}{4} & [3] & \multicolumn{4}{|c|}{$\begin{array}{l}\text { University } \\
\text { Hanol, Viet Nam }\end{array}$} & 3.27 & 8 & 6.9 \\
\hline & & \multicolumn{4}{|c|}{ Institute of Business Research. University of Economics Ho } & & & \\
\hline- & {$[--]$} & \multicolumn{4}{|c|}{ Ho Chl Mlinh Cty, Viet Nam } & 4.49 & 4 & 4 \\
\hline 5 & [5] & \multicolumn{4}{|c|}{$\begin{array}{l}\text { Institute of Public Pollcy Management (IPPM) } \\
\text { Henot, Viet Nam }\end{array}$} & 5.29 & 2 & 1.29 \\
\hline 6 & [9] & \multicolumn{4}{|c|}{$\begin{array}{l}\text { University of Economics, University of Danang } \\
\text { Dananz Vior Nam }\end{array}$} & 7.08 & 5 & 4.9 \\
\hline 7 & [6] & \multicolumn{4}{|c|}{$\begin{array}{l}\text { Business and Economlcs Research Group, Ho Chi Minh Clity } \\
\text { Open University } \\
\text { Ho Chi Minh aty, Viet Nam }\end{array}$} & 7.18 & 10 & 10 \\
\hline 8 & [7] & \multicolumn{4}{|c|}{$\begin{array}{l}\text { University Vietnam } \\
\text { Ho ChI Minh Cly, Viat Nam }\end{array}$} & 7.5 & 1 & 0.9 \\
\hline 9 & [8] & \multicolumn{4}{|c|}{$\begin{array}{l}\text { Development and Policies Research Center (Depecen) } \\
\text { Hanot, Viet Nam }\end{array}$} & 7.72 & 5 & 3.6 \\
\hline 10 & [10] & \multicolumn{4}{|c|}{$\begin{array}{l}\text { Vletnam } \\
\text { Ho Chi Minh Clyy, Vilet Nam }\end{array}$} & 10.4 & 4 & 4 \\
\hline 11 & [11] & \multicolumn{4}{|c|}{$\begin{array}{l}\text { National Economics University. } \\
\text { Hanot, viet Nam }\end{array}$} & 12.26 & 6 & 5 \\
\hline 12 & [12] & \multicolumn{4}{|c|}{$\begin{array}{l}\text { Department of Management of Sciences and Technology } \\
\text { Development. Ton Duc Thang University } \\
\text { Hochi Minh cly. Viet Nam }\end{array}$} & 15.12 & 3 & 2.08 \\
\hline
\end{tabular}

IDEAS is a RePEc $\widetilde{G}$ service hosted by the Research DIvislon $\widetilde{G}$ of the Federal Reserve Bank of St Louls $G$. RePEc uses blbllographic data respectlve publlshers. 
Danh sách này cũng cho thấy một thực tế: Các đơn vị có đầu tư và chính sách phát triển cho nghiên cứu mạnh mẽ, minh bạch và cụ thể đã và đang vươn lên trông thấy. Cụ thể đó là các đơn vị tiếng tăm: ĐH Kinh tế Tp.HCM, ĐH Kinh tế Đà Năng, ĐH Mở Tp.HCM, ĐH Fulbright. Cán bộ Trung tâm ISR cũng tự đánh giá, việc vẫn còn giữ ở tốp 5 của RePEc của Trung tâm có nhiều phần may mắn. Trong tương lai, khó khăn và thách thức sẽ ngày càng nhiều hơn với việc trụ lại ở nhóm này.[2]

Tuy nhiên, cũng có thêm thông tin phấn khởi bên lề. Bài trên Nature mẹ (151 tuổi) của ISR, sau khi xuất bản vào đầu tháng 6 , nay đã chỉ mục hóa vào CSDL ISI Web of Science trong tháng 72020, trong hình 3. Điều này giúp cho việc tracking lượng sử dụng thông tin khoa học và trích dẫn được thuận tiện và minh bạch. [3]

Cán bộ nghiên cứu ISR đều mong bài sẽ có thêm trích dẫn ISI WOS trong tương lai, và là một niềm vui trong lao động học thuật nhiều khó khăn, gian khổ. Một ngọn đèn thắp thêm tia sáng hy vọng cho công việc gian khó.

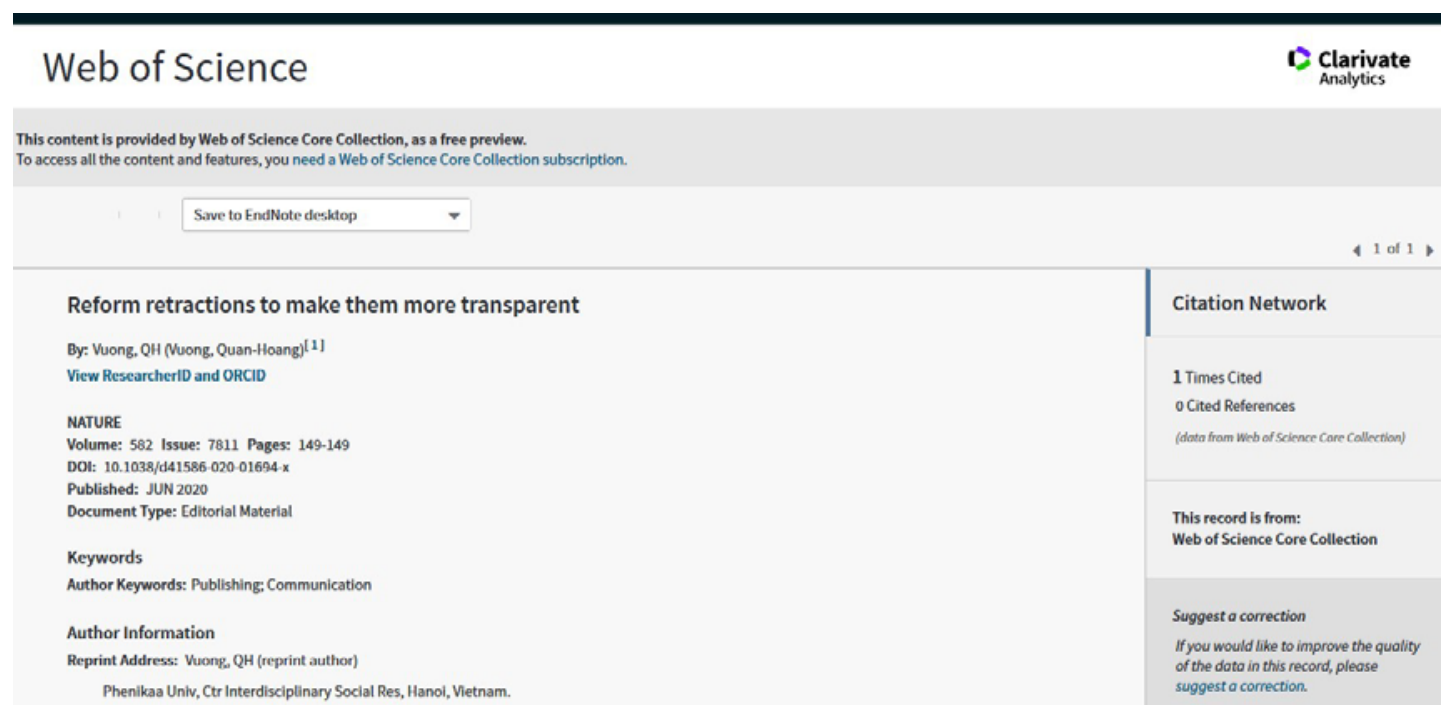

Hình 3. Bài ISR trên Nature đã chỉ mục hóa trong ISI Web of Science, dữ liệu Publons (ngày 5-8-2020).

Với nguồn lực vật chất vô cùng ít ỏi, số lượng nghiên cứu viên hạn chế, và khối lượng công việc lớn, đội ngũ đã và đang phấn đấu thực hành tốt tiêu chí đặt ra từ năm 2018 [4] về "tối thiểu hóa chi phí trong sản xuất khoa học". 
4 tháng còn lại của năm 2020 sẽ rất khó khăn. Tuy vậy, Trung tâm ISR vẫn giữ vững tinh thần và nỗ lực tối đa để có một năm lao động khoa học đầy xúc cảm, bất chấp những thách thức của thời cuộc và tình hình dịch bệnh kéo dài.

\section{Tài liệu tham khảo:}

[1] Springer Nature. (2020). SN Social Sciences (ISSN: 2662-9283). Editorial Board Member. Springer. https://www.springer.com/journal/43545 (truy cập: 7-8-2020)

[2] IDEAS RePEC. (2020). Economics Departments, Institutes and Research Centers in the World: Viet Nam. EDIRC RePEC. https://edirc.repec.org/vietnam.html (truy cập: 7-8-2020)

[3] Vuong, Q. H. (2020). Reform retractions to make them more transparent. Nature, 582(7811), 149-149. Available at: https://www.nature.com/articles/d41586-020-01694-x

[4] Vuong, Q. H. (2018). The (ir)rational consideration of the cost of science in transition economies. Nature Human Behaviour, 2(1), 5-5. Available at:

https://www.nature.com/articles/s41562-017-0281-4 